J.-M. TERPEREAU

SNCF

Direction de l'ingénierie

6 , avenue

François-Mitterrand 93574 La Plaine Saint-Denis jean-marc.terpereau@sncf.fr

Organisation des recherches dans le cadre du plan d'actions LGV Nord Caractérisation des limons sous différents états hydriques et sous sollicitations ferroviaires

Dans le contexte de recrudescence de fontis survenus au cours de l'hiver 2000-2001 particulièrement pluvieux (cf. inondations de la vallée de la Somme), et dans le cadre du rapport d'expertise du Conseil général des Ponts, RFF et la SNCF ont engagé des actions de recherche visant à : (i) caractériser le comportement des limons sous différents états hydriques et sous sollicitations ferroviaires, (ii) déterminer les mécanismes de rupture des fontis et leur vitesse d'évolution. Ces actions de recherche ont été lancées dès 2002 sous forme de projet élémentaire. Au fur et à mesure de la production des résultats, d'autres projets, ou phases de projet se sont greffés afin d'assurer auprès de la DTT (Direction des transports terrestres) la complétude des réponses apportées. Le présent article dresse le panorama d'ensemble de ces actions en rappelant, action par action, les attendus, et les principaux résultats obtenus.

Mots-clés : limon, état hydrique, chargement ferroviaire, fontis, mécanisme de rupture.

\title{
Organisation of researches
}

within the action plan

related to Northern LGV -

Characterisation of silt

at various hydric states

and under various

railway-based loadings 
(ii) determining, for the sinkholes, the failure mechanisms and its evolution rate. These actions were launched in 2002 by sub-projects. With the progress of the results production, other projects, or project phases are grafted in order to ensure the completeness of the answers for the DTT (Directorate of landing Transport). This paper presents the panorama of all these research activities by showing, action by action, the expected and the main results obtained.

Key words: silt, hydric state, railway-based loading, sinkholes, failure mechanism.

\section{Introduction}

Les intempéries de l’hiver 1993-1994 en Picardie et particulièrement sur certains secteurs de la ligne nouvelle à grande vitesse LGV Nord avaient conduit au déraillement à pleine vitesse d'une rame en décembre 1993 sur un fontis situé en voie d'environ $3 \mathrm{~m}$ de diamètre. Cet incident, sans dommage pour les personnes, avait mis en évidence la conception particulière ment favorable du TGV en cas de déraillement avec le boggie positionné au niveau de l'intercirculation entre deux voitures, gage d'une rigidité importante de la rame TGV. Celle-ci était en effet restée en ligne. Cela avait conduit à la réalisation d'un premier plan d'actions. Ainsi de nombreux chantiers d'injections sur des zones présentant des anomalies microgravimétriques confirmées par des sondages de reconnaissance et pouvant être mises en relation avec des cavités d'origine militaire avaient été réalisés.

Les intempéries exceptionnelles de l'hiver 2000 2001 d'intensité supérieure à celles de décembre 1993 ont provoqué des inondations sur une longue période de la Somme; de nombreuses communes situées dans la région Picardie étaient touchées par une recrudescence de fontis. Le réseau ferroviaire, de ce secteur avait subi également l'impact des intempéries, sous la forme de fontis de dimensions relativement limitées (1 à $2 \mathrm{~m}$ ), le plus souvent situés en limite de la plateforme ferroviaire à proximité des drainages latéraux. Au titre des mesures conservatoires, l'exploitation de la ligne s'est faite alors en conditions dégradées avec une vitesse réduite à $170 \mathrm{~km} / \mathrm{h}$ au lieu de $300 \mathrm{~km} / \mathrm{h}$ sur les tronçons concernés par ces fontis et cela en attente des travaux (injections et étanchement de fossés de plate-forme).

Bien que ces événements soient, de par leur taille et leur position, moins critiques que celui ayant conduit à l'incident de décembre 1993 (3 m en voie), les conséquences sur la régularité étaient fortes; il était donc nécessaire, au-delà des mesures utiles à la restitution en vitesse normale des conditions d'exploitation (comblement des cavités, injections complémentaires, amélioration de l'étanchéité des eaux de surface), d'en comprendre leur origine.

La mission confiée par le Conseil général des Ponts à M. Rat pour le compte de la Direction des transports terrestres avait conduit à identifier notamment le besoin d'améliorer les connaissances sur :

- les mécanismes à l'origine de ces fontis (en identifiant le rôle joué par la teneur en eau mais aussi l'influence éventuelle des vibrations induites clans le sol par les circulations TGV;

- les méthodes d'investigation permettant de caractériser ces phénomènes.
Un panorama de ces différentes actions est proposé dans le présent article. On note qu'il a fallu faire appel à des niveaux élevés de compétences dans des domaines aussi multiples que variés telles la géologie du quaternaire, la mécanique des sols non saturés, la dynamique des sols, la modélisation du passage d'une circulation, etc. In fine, il a fallu aussi se confronter « au terrain s, et à travers une campagne d'investigations géotechniques particulièrement élaborée, faire le lien entre la compréhension de phénomènes identifiés au laboratoire et résultats d'une campagne de reconnaissance.

Le tableau I présente les rapports de recherche réalisés par les différents acteurs dans le cadre spécifique du projet.

\section{2 \\ Panorama des actions 9.1. \\ Étude bibliographique - Identification des compétences}

Dans un premier temps, une importante recherche bibliographique a été entreprise dans les domaines de la mécanique des sols non saturés et de la dynamique des sols. Elle a abouti début 2003, à travers la production d'une liste bibliographique riche de 126 références classées par thème. La caractérisation géologique, préalable à toute action s'est imposée. Sous le terme générique de limons des plateaux tel que décrit dans les cartes géologiques du Nord de la France se cache l'extrême diversité des loess [2] et [3] :

« Les lœss sont des limons d'origine éolienne caractéristiques des environnements périglaciaires du quaternaire (depuis 2,4 Ma). Ils se localisent essentiellement entre 30 et $60^{\circ}$ de latitude Nord, en marge des grands inlandsis et des zones désertiques (Chine). Les alternances loess-paléosols constituent des séquences lœessiques dont l'épaisseur de l'ordre de 5 à $40 \mathrm{~m}$ en Europe occidentale et centrale, peut localement dépasser $200 \mathrm{~m}$ en Chine où ils correspondent à environ 2,4 Ma. Actuellement l'étude pluridisciplinaire de ces séquences est un des moyens privilégiés de reconstitution de l'enregistrement paléo-climatique en domaine continental. Au sein de ces séquences, les lœess typiques sont constitués par des silts quartzeux (fraction dominante 10-50 $\mu \mathrm{m}$ ), calcaires, extrêmement bien classés, pauvres en sable, non stratifiés, et qui possèdent une structure isotrope fortement poreuse et métastable. D'un point de vue géotechnique, ce sont des matériaux effondrables, extrêmement sensibles aux variations d'humidité, à la 


\section{Tit:}

Caractérisation des limons - Modélisation numérique de la réponse dynamique d'une plate-forme ferroviaire fondée sur limons

Caractérisation des limons - Étude du comportement des limons non saturés soumis aux vibrations ferroviaires

140.855

Caracterisation des limons - Application de la méthode d'analyse spectrale des ondes de surface sur le site SNCF de Beugnâtre

Caractérisation des limons - Mise en place capteurs - Suivis

\section{SEGG}

Caractérisation des limons - Compte rendu : Installation de sondes d'humidité en forage

Caractérisation des limons - Auscultation par diagraphies nucléaires
$05 / 12 / 2002$

ENPC

- rapport Lot 1 du 26/07/2003

- rapport Lot 2 du 26/09/2003

- rapport Lot 3 du 17/05/2004 clu 14/10/2003 et rapport complémentaire février 2004

BRGM, décembre 2002

Sols Mesures, 24/07/2003

LROP, dossier n 40768-1 du 5/07/2004

compaction et aux vibrations. Dans le Nord-Ouest de l'Europe, où ils constituent une zone plus ou moins continue de la Bretagne à la vallée de Rhin, l'analyse détaillée de la séquence du Pléistocène supérieur $(128 / 15 \mathrm{ka}, \mathrm{BP})$ permet de mettre en évidence une succession pédosédimentaire complexe au sein de laquelle les lœss carbonatés sont caractérisés d'une courte période centrée autour du dernier maximum glaciaire (30-15 ka, BP). »)

Il faut également noter l'importance de l'école russe à travers les publications d'Abelev et Abelev [1]. On mentionnera également les travaux de Gibbs et Bara [7].

Il faut souligner la contribution de Cui et al. [4] à [6] s'intéressant, suite au déraillement de décembre 1993, à la caractérisation au laboratoire sous les aspects d'effondrabilité des lœess rencontrés sur le tracé du TGV Nord.

A l'issue de cette phase de bibliographie, il est apparu clairement que, si le domaine du comportement des lœss sous différents états hydriques était richement développé dans la littérature avec les écoles russe, chinoise et américaine, le couplage avec le domaine vibratoire est lui très peu exploré hormis en tant que vibration générée en phase travaux pour éliminer un risque d'effondrement (vibroflottation en phase chantier). En particulier, le comportement des lœss sous différents états hydriques et sous vibration ferroviaire n'a jamais été appréhendé.

Cette première étape a permis d'identifier les meilleures compétences disponibles dans les domaines vibratoires (Géodynamique et Structures) ainsi qu'en mécanique des sols non saturés (ENPC) pour réaliser les études présentées ci-après.

Un (" macrozonage » de la distribution des lœss le long de la ligne a pu être établi à partir de l'exploitation des campagnes de reconnaissances réalisées pour la réalisation de la Ligne à Grande Vitesse [9]. Une intégration dans le SIG (Système d'informations géographique) est prévue.

\section{2}

\section{Étude paramétrique du comportement d'une plate-forme ferroviaire et du sol support sous sollicitations type TGV (Géodynamique et Structures)}

Au vu du constat précédent, une première étude a été entreprise afin de fournir, pour différentes conditions de site, des ordres de grandeur des sollicitations générées par les circulations ferroviaires. Ces modélisations étaient un préalable à la réalisation des essais en laboratoire (ENPC) afin de définir les protocoles de réalisation de ces essais. Cette étude a été confiée à Géodynamique et Structures, bureau d'études spécialisé en dynamique des sols et ayant déjà une expérience du comportement des plate-formes ferroviaires sous circulation à grande vitesse, tant en mesures in situ, qu'en modélisation [10] et [11]. Ainsi le modèle utilisé MISS 2.5 D avait été préalablement étalonné sur la LGV Nord avec des mesures de terrain dont disposait Géodynamique et Structures. Les résultats de cette étude ont également permis d'identifier des zones proches de la plate-forme ferroviaire (zone de fossés ou de collecteurs drainants) qui sont affectées par des combinaisons de sollicitations défavorables (déconfinement et cisaillement cyclique) au passage des circulations.

Ces niveaux de sollicitations ont ensuite été validés à l'occasion de campagnes de mesures réalisées sur le site test de Beugnâtre.

\section{Campagne d'essais en laboratoire (ENPC)}

Trois phénomènes ont pu être identifiés et quantifiés à l'issue de la campagne d'essais en laboratoire :

- l'effondrement, tassement immédiat au mouillage sous charge prédéfinie, mécanisme significatif d'amplitude limitée (2 à $5 \%$ ) aboutissant à une augmentation de la stabilité du squelette granulaire ; 
- l'endommagement, fatigue et réorganisation du squelette sous l'influence des circulations ferroviaires répétées et pour un état hydrique donné, mécanisme significatif d'amplitude négligeable dans des états hydriques normaux ;

- la liquéfaction, perte quasi totale de la résistance mécanique du sol saturé sous sollicitations ferroviaires TGV, mécanisme significatif avec déformations importantes (déformation axiale $\approx 20 \%$ ) générant un état instable.

On note cependant que suivant les recommandations de l'Association française du génie parasismique (AFPS 90), les lœss rencontrés sur le tracé du TGV Nord ne sont pas considérés comme liquéfiables.

\section{Campagne d'essais de reconnaissance in situ, site expérimental de Beugnâtre}

L'objectif de la campagne menée sur le site expérimental de Beugnâtre (Fig. 1) était de disposer d'une banque de données de la réponse des différentes techniques de reconnaissances en conditions d'état hydrique dite ( courant ) et sous des teneurs en eau proches de la saturation. L'objectif final était de pouvoir proposer une campagne de reconnaissance spécifique au contexte géotechnique de la LGV. En fonction des évolutions du périmètre du projet, un objectif supplémentaire a ensuite été de fournir les données entrantes à un outil développé par l'ENPC permettant de qualifier le niveau d'aléa (cf. paragraphe spécifique développé plus loin). Une campagne de reconnaissance a été réalisée en juillet 2003 par l'entreprise FUGRO. Le LROP est intervenu également pour la réalisation des diagraphies nucléaires. Le BRGM a fourni pour sa part une caractérisation des lœess par méthode géophysique SASW (ondes de surface). Une liste des méthodes de reconnaissance déployées lors de cette campagne est présentée dans le tableau II ; chaque méthode a été testée sous son état hydrique naturel et sous un état proche de la saturation (plots d'essais spécifiques).
TABizau II Liste des méthodes déployées sur le site expérimental de Beugnâtre.

List of the methods tested at the experimental site of Beugnâtre.

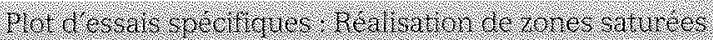

Sondage carotté

Sondages pressiométriques

Sonclages au pénétromètre dynamiques

Sondages au pénétromètre statique

(avec pointes piézocône, sismique, pressiométrique et vidéo)

Sondages SPT

Sondages destructifs

Essais scissométriques

Essais au Dilatomètre plat Marchetti (DMT)

Mesures par diagraphies nucléaires

Un exemple de deux sondages pénétrométriques (dynamique et statique) est reporté sur la figure 2 ; il est aisé de remarquer que la résistance mesurée à l'aide de cet appareil est fortement altérée avec une augmentation de la teneur en eau du sol; cette chute de la résistance est limitée aux horizons compris entre 2 et $3 \mathrm{~m}$ de profondeur (horizons lœessiques les plus sensibles).

Ce chantier a également permis de comparer les réponses des enregistrements de paramètres en sondage avec 8 paramètres (VA, PI, PO, PR, CR, VR, débit, percussion réfléchie). Pour ce site parfaitement caractérisé, les méthodes ou moyens suivants ont pu être comparées :

- rotation pure ou rotopercussssion ;

- diamètre 64 ou 89 ;

- fluide de forage eau, GSP, air.

De façon concertée avec l'entreprise de sondages, une mise en cualité assez poussée de l'atelier de forage a été appliquée. A titre d'exemple, l'ensemble des capteurs sur la machine a été vérifié d’un point de vue métrologie. Alors qu'usuellement il est privilégié dans le cadre de la détection de cavités, le recours à la rota-

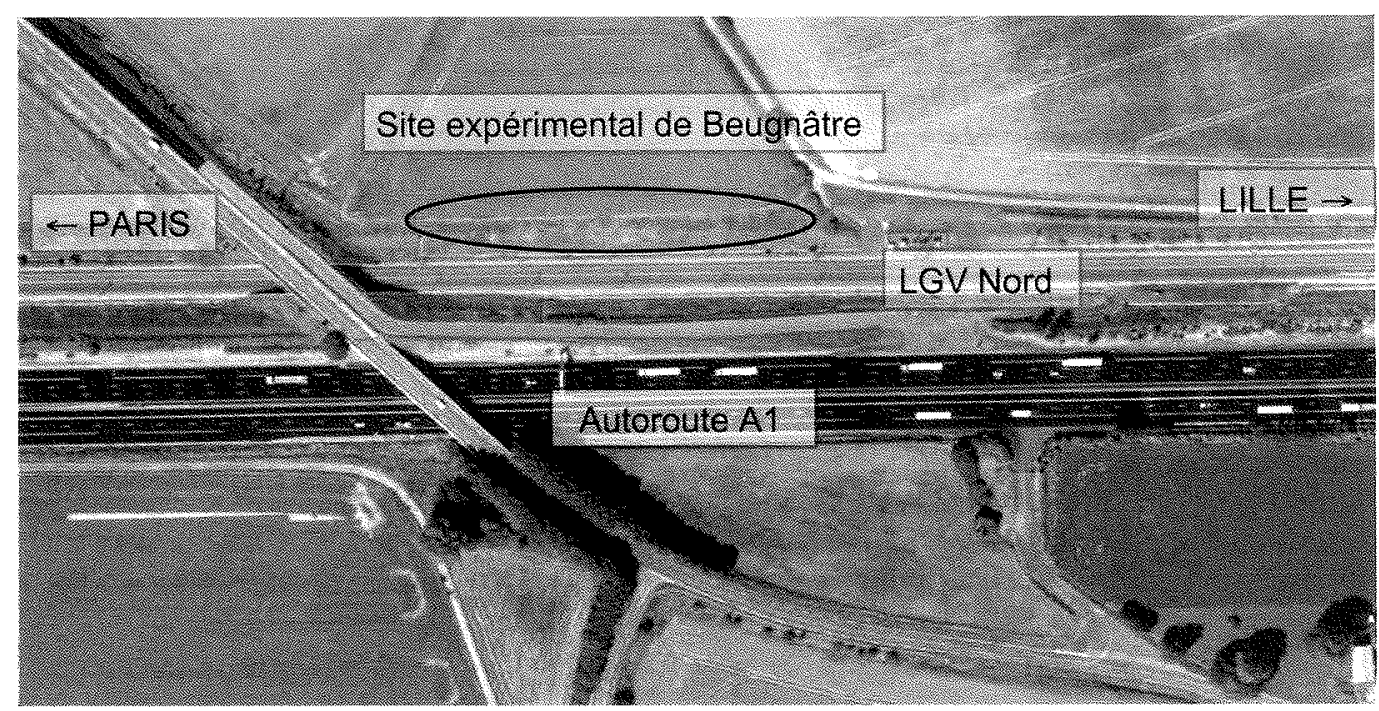

FG. 1 Vue aérienne du site expérimental de Beugnâtre (km 141). Photographie prise lors du vol infrarouge de la ligne en septembre 2001.

Aerial view of the experimental site of Beugnattre (km 141). Photography taken during the infrared flight of the line in September, 2001. 

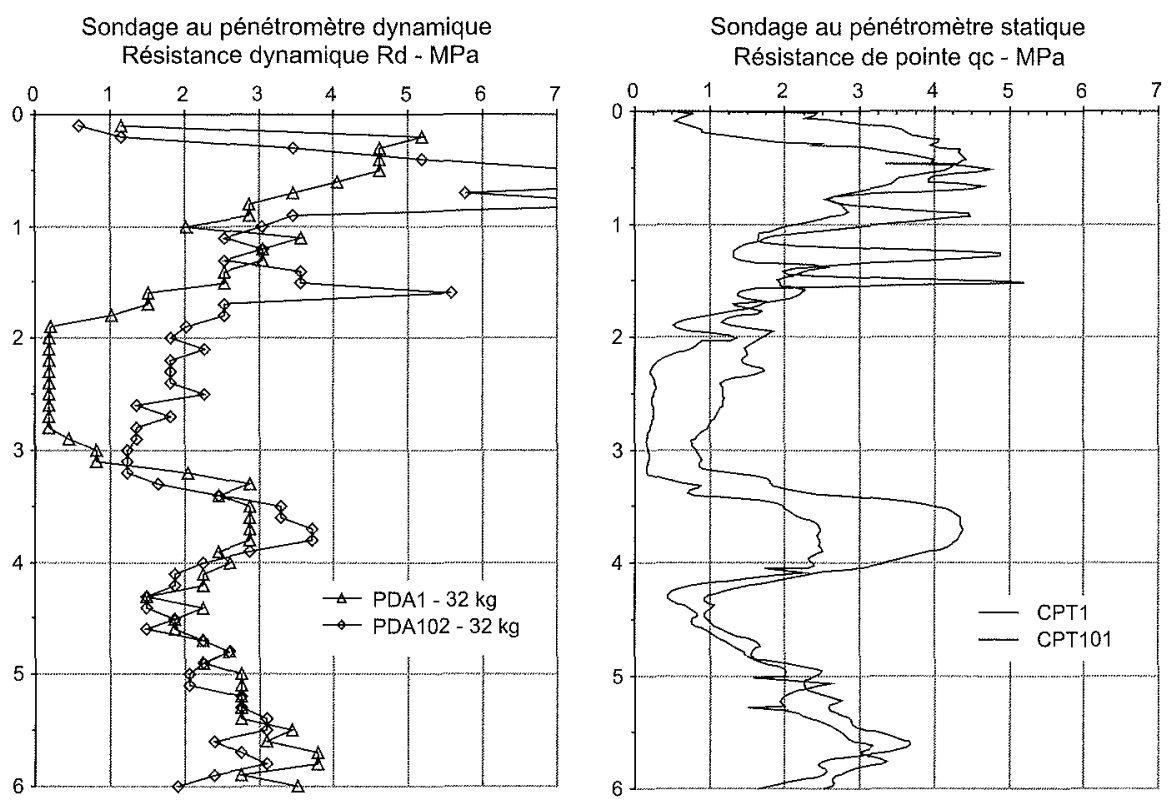

16. 2. Exemple de l'influence de l'état hydrique (saturation de la tranche 0 à $5 \mathrm{~m}$ ) sur la réponse de sondages au pénétromètre dynamique et au pénétromètre statique (Campagne FUGRO 2003). Courbe PDA102-CPT101 (décalée à droite) : sondage sous état hydrique naturel ; courbe PDA1-CPT1 (décalée à gauche) : sondage sous état proche de la saturation.

Example of the influence of hydric state (saturation of the trenche from 0 to $5 \mathrm{~m}$ ) by the response of the investigation using dynamic penetrometer and static penetrometer (Campain FUGRO 2003). Curve PDA102CPT101 (rightwards) : investigation at natural state ; curve PDA1-CPT 1 (leftwards) : investigation at a state close to saturation.

tion pure, un forage dans un diamètre le plus important possible et l'utilisation de fluide non mouillant, d'excellents résultats ont été obtenus en forage à l'eau diamètre 64 .

Les premières conclusions qui peuvent être dressées sont les suivantes :

- le qualificatif de (c décomprimé » utilisé fréquemment pour qualifier les limons ne correspond généralement pas à un état permanent du sol (hormis à l'aplomb d'une tranchée militaire par exemple) mais dépend fortement de sa teneur en eau ;

- le mode de prélèvement d'échantillon affecte les résultats des essais en laboratoire; après comparaison la méthode la plus appropriée est le prélèvement de blocs en place dans une tranchée ;

- vis-à-vis du phénomène de liquéfaction, la campagne de reconnaissance type retiendra plusieurs méthodes parmi les suivantes:

- sondage carotté couplé à des essais d'identification (limites d'Atterberg, mesure de la teneur en eau),

- géophysique par méthode des ondes de surface (SASW),

- sondage au pénétromètre statique,

- sondage au pénétromètre dynamique,

- sondage destructif avec enregistrement de paramètres,

- sondage avec diagraphie pour mesures de densité, dilatomètre Marchetti ;

- vis-à-vis de l'objectif assigné, certaines techniques de reconnaissance se sont révélées peu pertinentes :
- sondage scissométrique (mesure impossible dans un état hydrique normal),

- sondage pressiométrique (intégration de couches d'épaisseur trop importante)

D’une manière générale, étant donnée la difficulté de diagnostic du potentiel de liquéfaction, toutes les campagnes « monométhode », ou sans mise en qualité suffisante des ateliers, sont à proscrire.

\section{5}

\section{Campagne d'instrumentation du site de Beugnâtre}

L'objectif de l'instrumentation déployée sur le site expérimental de Beugnâtre était de :

- vérifier expérimentalement les ordres de grandeurs des vibrations fournies par les modélisations réalisées par Géodynamiques et Structures et utilisés dans le cadre de la définition des protocoles pour les essais en laboratoire (campagne de l'ENPG) ;

- estimer les paramètres dynamiques (modules de cisaillement (G) et modules d'Young (E)) et leurs évolutions suivant les variations d'état hydrique;

- suivre en continu les variations d'état hydrique dans le temps et à différentes profondeurs en parallèle avec le suivi pluviométrique.

Le site de Beugnâtre a fait l'objet d'une instrumentation en sondes d'état hydrique (Fig. 3), et en capteurs de vibrations : accéléromètres et géophone triaxiaux (Fig. 4). Les éléments du suivi (débuté depuis 


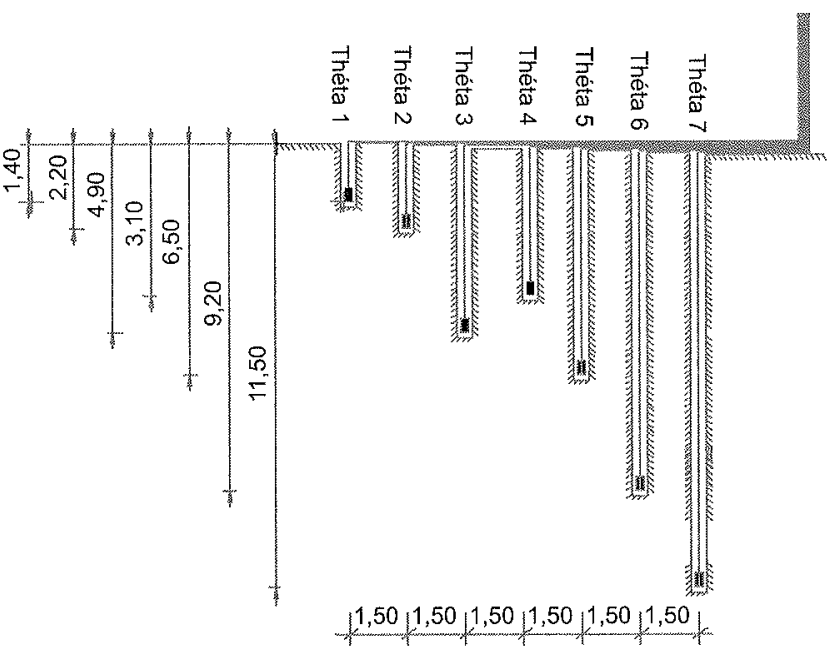

1.6. 3 Implantation des sondes de teneurs en eau en crête de talus, site de Beugnâtre.

Installation of water content sensors on top of the slope, site of Beugnâtre. mars 2004) nous indiquent des variations limitées de la teneur en eau volumique (période climatique relativement sèche, voir Figs. 5 et 6 ).

Les résultats du suivi de l'instrumentation (Figs. 7 et 8) ont permis de montrer que les ordres de grandeur des vibrations prises en compte à l'issue des modélisations sont confirmés en termes d'intensité et de contenu fréquentiel ; ces mesures sont par ailleurs cohérentes avec celles réalisées dans le cadre du programme européen Supertrack (Sustained Performance of Railways Track) qui s'intéresse notamment au comportement dynamique de la voie ballastćc [12].

Les valeurs prises en compte pour la réalisation des essais en laboratoire ont donc été validées a posteriori.

Un autre objectif était de suivre les variations des modules dynamiques dans le temps et en fonction des variations d'état hydrique ; cet ambitieux volet n'a pu être mené à son terme. La finalité à terme aurait été de disposer in situ d'un dispositif avertisseur d'une évolution préjudiciable des conditions de portance de la plate-forme.

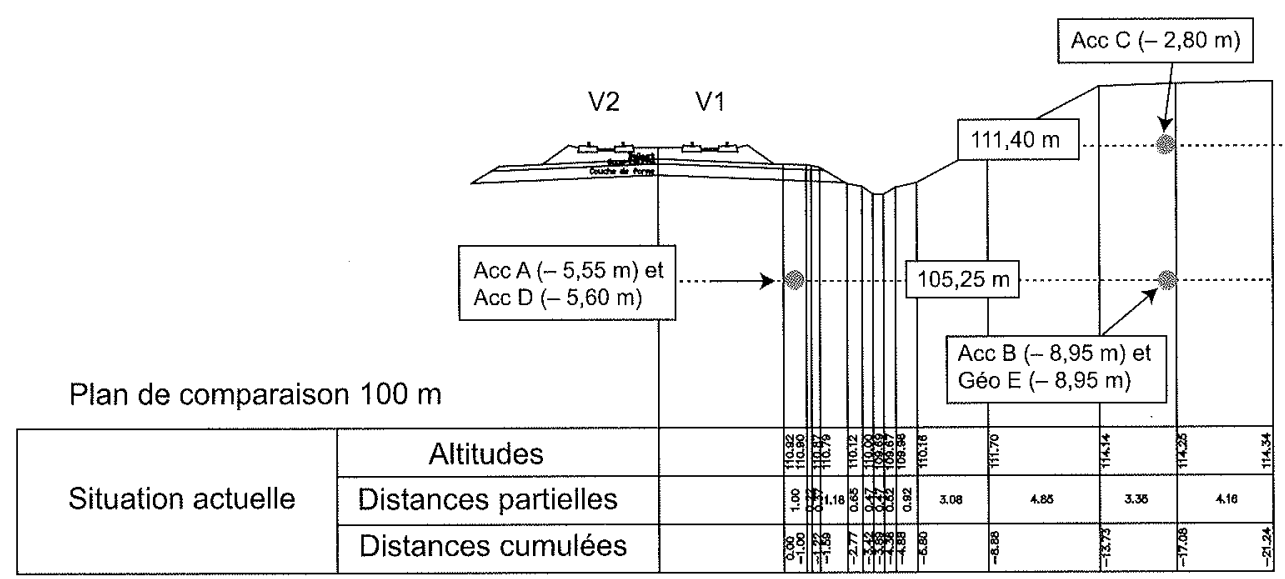

Ho. 4 Implantation des capteurs de vibration. Installation of vibration sensors.

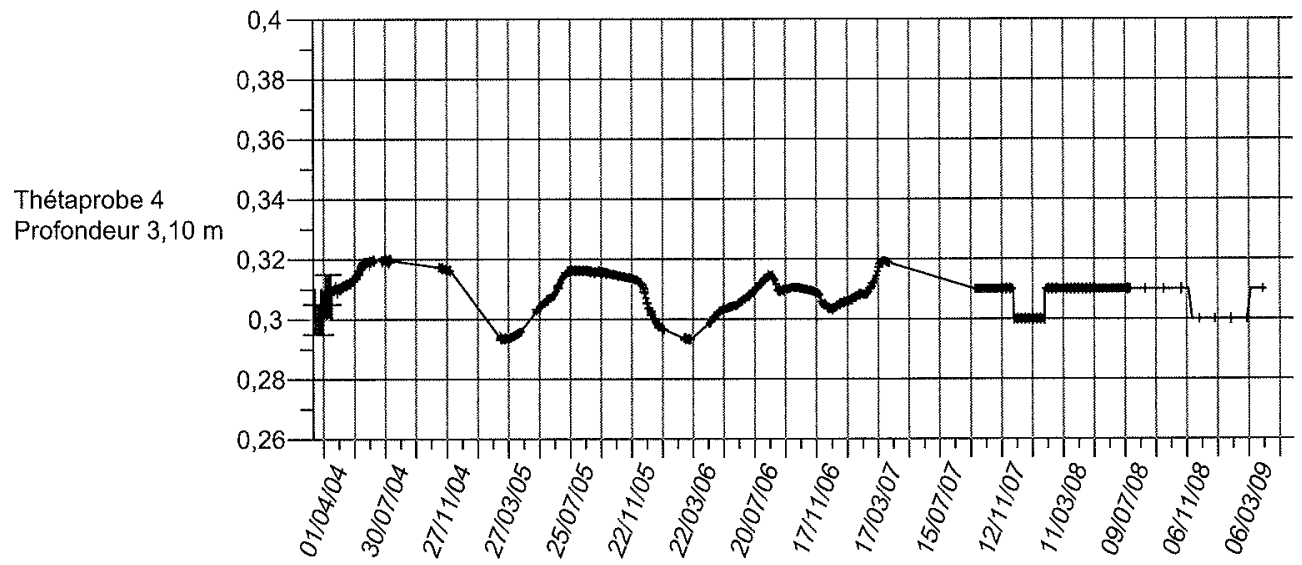

FG. Sariation des teneurs en eau volumiques en fonction du temps (capteur situé à $3,1 \mathrm{~m}$ de profondeur).

Variation of volumetric water content with time (sensor situated at $3.1 \mathrm{~m}$ depth). 


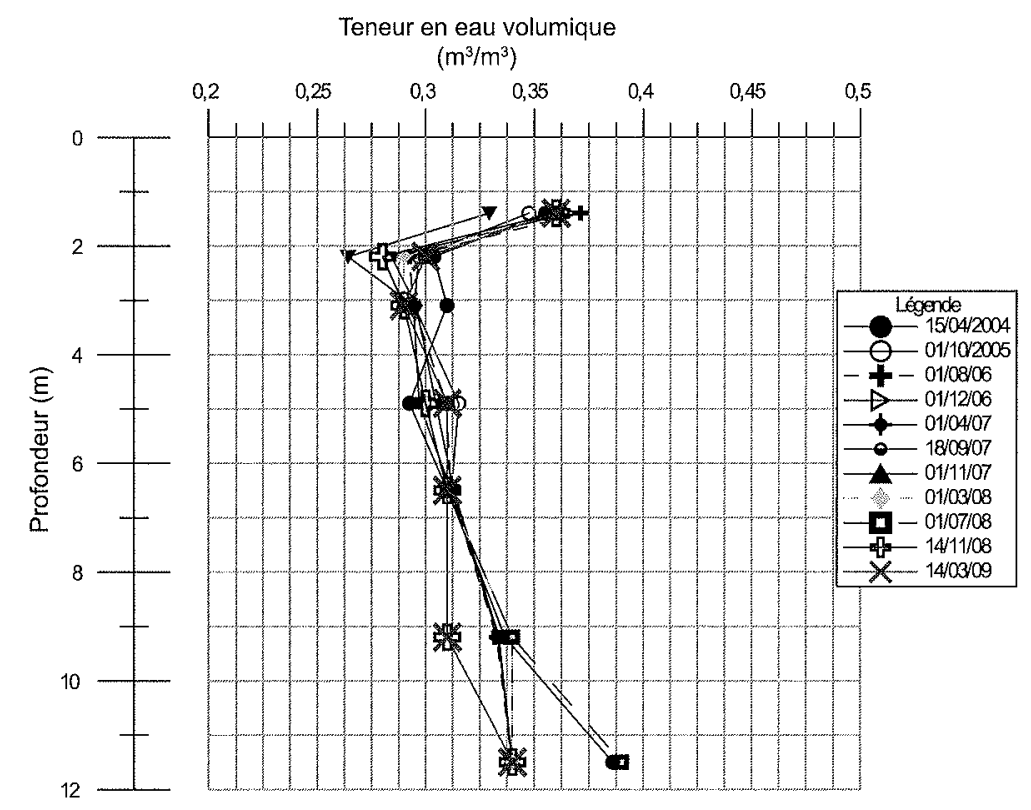

Ic. 6 Évolution des profils hydriques entre octobre 2005 et janvier 2006.

Evolution of hydric profiles from between October 2005 and January 2006.
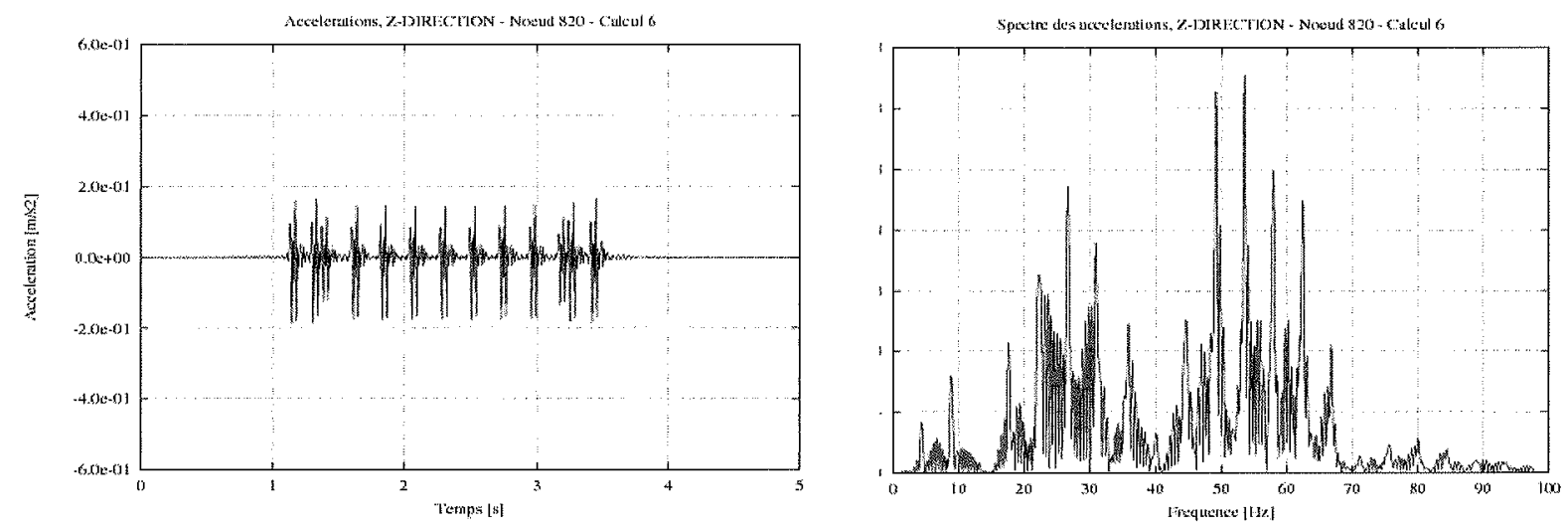

Résulats des modélisations (d'après Géodynamique et Structures).

Représentation des accélérations à $4 \mathrm{~m}$ de l'axe de la voie et à $6 \mathrm{~m}$ de profondeur sous le TN.

A gauche ; accélérations en fonction du temps ; à droite : contenu fréquentiel.
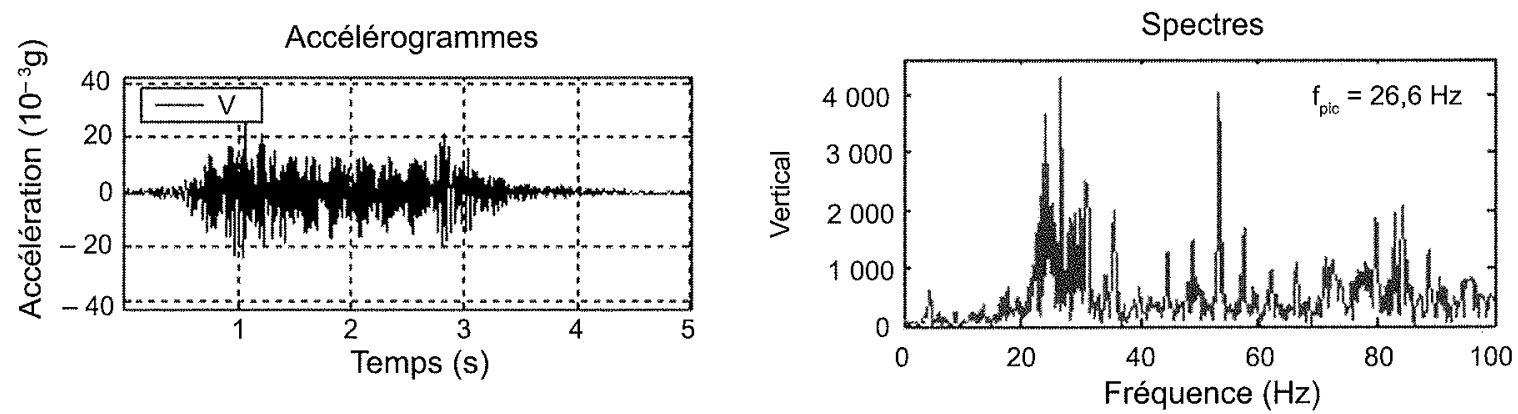

Résultats expérimentaux (d'après SEGG).

Représentation des accélérations à $4 \mathrm{~m}$ de l'axe de la voie et à $5,55 \mathrm{~m}$ de profondeur sous le TN (accéléromètre $\mathrm{A}$ ).

A gauche : accélérations en fonction du temps ; à droite : contenu fréquentiel.

FG. 7 Mesures de vibrations. Comparaison des modélisations et des résultats expérimentaux. Vibrations measurements. Comparison between modelling and experiment. 


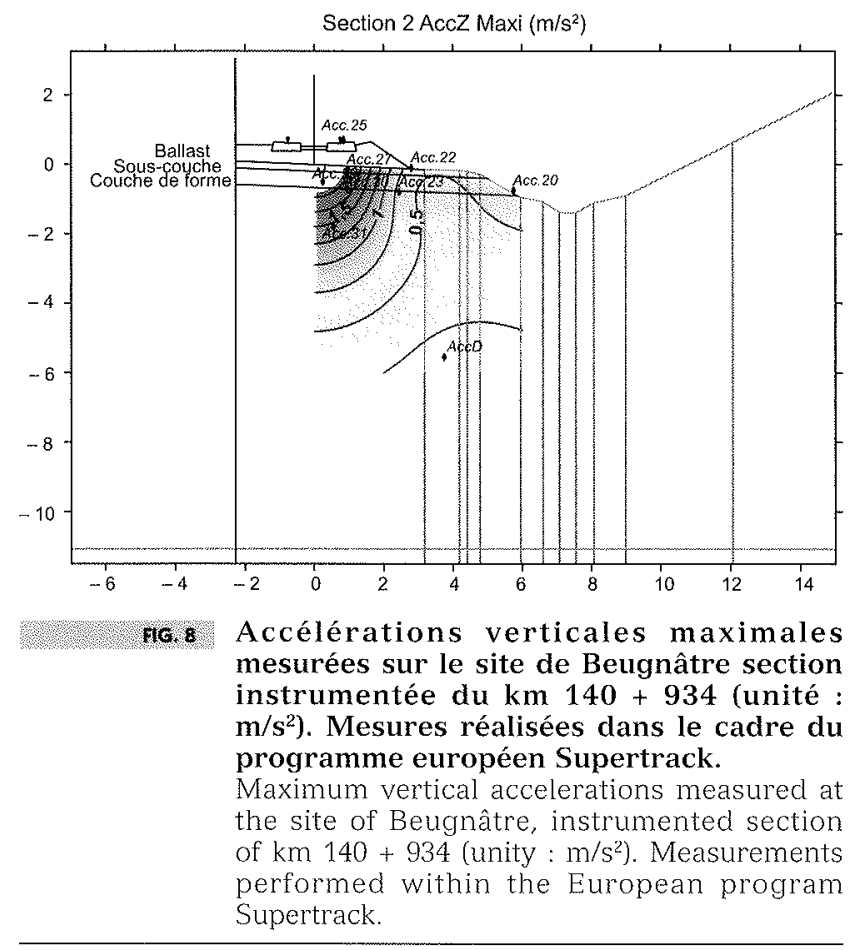

\section{3}

\section{Conclusion}

Ces différents projets ont été conduits en gardant à l'esprit à la fois, la recherche d'une explication à des phénomènes physiques observés, le cadre de la sécurité des circulations ferroviaires, la recherche de solutions de surveillance innovantes (actuellement les principes de surveillance sont essentiellement basées sur des déclenchements de tournées hélicoptère sur la base de dépassement de seuils pluviométriques) ; le tout dans un cadre budgétaire optimisć.

Globalement ces recherches n'ont pas servi simplement à caractériser les phénomènes d'instabilité affectant les lœess. Elles ont permis de fournir un argumentaire étayé au propriétaire de l'infrastructure (RFF) notamment pour engager des actions préventives d'étanchement de fossés de plate-forme. Elles ont bien entendu également servi à répondre aux interrogations formulées par la DTT.

Enfin les derniers développements, non exposés dans le cadre du présent article consistent à (i) quantifier l'aléa en cas de saturation ultérieure du sol, à partir des résultats d'une campagne d'investigations type, et (ii) disposer d'une méthode alternative aux injections par des coulis (imprégnation).

\section{Bibliographie}

[1] Abelev Y.M., Abelev M.O.Y. - Bases de I'élaboration des projets et de la construction sur les sols lœessiques affaissables. Lavoisier. Techniques \& Documentation, Paris (traduction française J.-P. Magnan), 1986, $787 \mathrm{p}$.

[2] Antoine P. - Les loess en France et dans le Nord-Ouest européen. Revue française de géotechnique, $n^{\circ}$ 99, 2002, p. 3-21.

[3] Antoine P., Cattt J., Somme J. - The Loess and Coversands of Northern France and Southern England. Journal of Quaternary Science, 18, 2003, p. 309-318.

[4] Cui Y.-J., Delage P., Alzoghbi P. - Étude du comportement des lcess sur le tracé du TGV Nord. Rapport de recherche ENPC, 1997
[5] Cui Y.-J., Delage P., Schlosser F., Wojnarowicz M. - Étude du comportement volumique d'un lœess du Nord de la France. Geotechnical Engineering for Transportation Infrastructure, Ed. Balkema, Rotterdam, 1999, p. 337-342.

[6] Cui Y.-J., Delage P., Durand F, Schlosser F., Wojnarowicz M. - Comportement mécanique des lœss sur le tracé du TGV Nord. Proc. 11th Eur. Conf. On Soil Mechanics and Foundation Engineering, vol. 7, 1995, p. 45-50.

[7] Gibbs H.J., Bara J.P. - Predicting surface subsidence from basic soil tests. Special technical publication, $\mathrm{n}^{\circ}$ 322, ASTM, 1962, p. 231-247

[8] Picoux B. - Etude théorique et expérimentale de la propagation dans le sol des vibrations émises par un trafic ferroviaire. Thèse de doctorat de l'École centrale de Nantes/université de Nantes, 2002.

[9] Terrasol - Localisation des lœess sur la ligne du TGV Nord. Rapport Terrasol $n^{\circ}$ 1994.030AC/02, 1998

[10] Ropers F., Pecker A. - Remblai de Ressons - LGV Nord. Contribution à l'analyse du comportement dynamique de la plate-forme, note $12^{\circ} 1$. Rapport de Géodynamique et Structures, 2000.

[11] Ropers F, Pecker A. - Remblai d'Hat tencourt - LGV Nord. Contribution à l'analyse du comportement dynamique de la plate-forme, note $n^{\circ} 2$. Rapport de Géodynamique et Structures, 2000.

[12] Supertrack G1RD-CT-2002-00777 Final Report, December 2005. 